\title{
RETRACTION
}

\section{Retraction: Polarization-entangled photons produced with high-symmetry site-controlled quantum dots}

Arun Mohan, Marco Felici, Pascal Gallo, Benjamin Dwir, Alok Rudra, Jérôme Faist and Eli Kapon

Nature Photonics 4, 302-306 (2010); published online: 7 March 2010; retracted online: 16 October 2012.

In this Letter, we claimed the generation of entangled photons from highly symmetric site-controlled pyramidal quantum dots. Apart from the observation of isotropic emission and the measurement of a vanishing fine-structure splitting of $0 \mu \mathrm{eV} \pm 2 \mu \mathrm{eV}$, a fidelity of $>0.5$ was obtained by using the density matrix derived from quantum state tomography of 16 different polarization-resolved correlation measurements. After further investigation of the second-order correlation functions, $g^{(2)}(\tau)$, major errors were found and the best fidelity found is now 0.45 for a gate width of $0.225 \mathrm{~ns}$. As a result, we can no longer claim emission of polarization-entangled photons. We therefore wish to retract this Letter and sincerely apologize for any adverse consequence that may have resulted from the paper's publication. 